\title{
3-Bromopyruvate and sodium citrate target glycolysis, suppress survivin, and induce mitochondrial-mediated apoptosis in gastric cancer cells and inhibit gastric orthotopic transplantation tumor growth
}

\author{
TING-AN WANG ${ }^{*}$, XIAO-DONG ZHANG ${ }^{*}$, XING-YU GUO, SHU-LIN XIAN and YUN-FEI LU \\ Department of Gastrointestinal and Gland Surgery, The First Affiliated Hospital of \\ Guangxi Medical University, Nanning, Guangxi 530021, P.R. China
}

Received September 23, 2015; Accepted November 17, 2015

DOI: $10.3892 /$ or.2015.4511

\begin{abstract}
Glycolysis is the primary method utilized by cancer cells to produce the energy (adenosine triphosphate, ATP) required for cell proliferation. Therefore, inhibition of glycolysis may inhibit tumor growth. We previously found that both 3-bromopyruvate (3-BrPA) and sodium citrate (SCT) can inhibit glycolysis in vitro; however, the underlying inhibitory mechanisms remain unclear. In the present study, we used a human gastric cancer cell line (SGC-7901) and an orthotopic transplantation tumor model in nude mice to explore the specific mechanisms of 3-BrPA and SCT. We found that both 3-BrPA and SCT effectively suppressed cancer cell proliferation, arrested the cell cycle, induced apoptosis, and decreased the production of lactate and ATP.3-BrPA significantly reduced the glycolytic enzyme hexokinase activity, while SCT selectively inhibited phosphofructokinase-1 activity. Furthermore, 3-BrPA and SCT upregulated the expression of pro-apoptotic proteins (Bax, cytochrome $c$, and cleaved caspase-3) and downregulated the expression of anti-apoptotic proteins (Bcl-2 and survivin). Finally, our animal model of gastric cancer indicated that intraperitoneal injection of 3-BrPA and SCT suppressed orthotopic transplantation tumor growth and induced tumor apoptosis. Taken together, these results suggest that 3-BrPA and SCT selectively suppress glycolytic enzymes, decrease ATP production, induce mitochondrial-mediated apoptosis, downregulate survivin, and inhibit tumor growth.
\end{abstract}

Correspondence to: Professor Yun-Fei Lu, Department of Gastrointestinal and Gland Surgery, The First Affiliated Hospital of Guangxi Medical University, 6 ShuangYong Road, Nanning, Guangxi 530021, P.R. China

E-mail: doctorlife@126.com

*Contributed equally

Key words: apoptosis, gastric cancer, 3-bromopyruvate, sodium citrate, gastric orthotopic transplantation tumor
Moreover, an intraperitoneal injection is an effective form of administration of 3-BrPA and SCT.

\section{Introduction}

Gastric cancer, one of the most common cancer types, is the second leading cause of cancer-related deaths worldwide. In recent years, the prognosis of gastric cancer patients has improved owing to the combined application of surgical techniques and chemotherapies. Nonetheless, the 5-year survival rate remains low $(1,2)$. Thus, new strategies to overcome gastric cancer are urgently required.

Compared with normal cells, most cancer cells preferentially depend on glycolysis to produce adenosine triphosphate (ATP) for growth and proliferation (3). This phenotype, referred to as 'aerobic glycolysis', was first observed by Warburg (4). Furthermore, our previous experiments and other researches have confirmed that gastric cancer utilizes glycolysis to meet the energy demand (5-7). ATP reduction by inhibiting glycolysis induces DNA degradation and cell apoptosis in tumors $(8,9)$. Therefore, glycolysis suppression may be an appropriate target for inhibiting cancer cell growth and/or inducing apoptosis. Hexokinase (HK), phosphofructokinase 1 (PFK-1), and pyruvate kinase (PK) are crucial enzymes that regulate the rate of glycolysis (10-13). HK binds to the mitochondrial membrane to catalyze the first rate-regulating step of glycolysis, and also enhances cell proliferation and suppresses apoptosis (14). PFK-1 catalyzes the conversion of fructose-6-phosphate and ATP into fructose 1,6-bisphosphate and ADP, while PK converts phosphoenolpyruvate and ADP into ATP in the glycolysis pathway. Therefore, if suppression of glycolysis can induce cancer cell growth inhibition and/or apoptosis, then HK, PFK-1 and PK may be potential targets for developing novel anticancer agents (15).

Both glycolysis and apoptosis have been regarded as independent pathways crucial for tumor cell survival $(16,17)$. Apoptosis can be activated through the endoplasmic reticulum, death ligand, and mitochondrial pathways $(18,19)$. The mitochondrial pathway is regulated by the activity of pro- and anti-apoptotic members in the Bcl-2 family. Pro-apoptotic proteins, such as Bax, increase mitochondrial membrane 
permeability, causing the secretion of cytochrome $c$ (Cyt-C), which activates the caspase cascade and initiates cell apoptosis (20-22). On the other hand, Bcl-2 prevents the accumulation of Cyt-C and the activation of the caspase cascade by stabilizing mitochondrial permeability, thereby inhibiting apoptosis $(23,24)$. Thus, any agent with the ability to regulate Bcl-2 family members and/or caspases in tumor cells may induce mitochondrial-mediated apoptosis. Similarly, survivin is a member of the inhibitor of apoptosis (IAP) family that is overexpressed in a variety of human cancers $(25,26)$, and may be an important anticancer target for gastric tumors.

Bromopyruvate (3-BrPA) is an alkylating agent that inhibits tumor growth and induces cell apoptosis by a variety of biochemical mechanisms (27-29). In our previous study, we found that both 3-BrPA and sodium citrate (SCT) can inhibit cancer cell proliferation in vitro $(6,7)$. However, their underlying inhibitory mechanisms require further investigation. In the present study, we developed an orthotopic transplantation tumor model in nude mice using human gastric cancer cells. We chose this animal model as the biological behaviors of the gastric orthotopic transplantation tumor model are more similar to the processes of growth and metastasis of human gastric cancer than the conventional xenograft models (subcutaneous or intraperitoneal injection of cancer cells) $(30,31)$. We aimed to explore the specific inhibitory mechanisms of 3-BrPA and SCT and their effects on apoptosis-related genes in gastric cancer. Moreover, we aimed to determine whether an intraperitoneal injection is an effective form of administration of 3-BrPA and SCT.

\section{Materials and methods}

Reagents. RPMI-1640 medium and fetal bovine serum (FBS) were purchased from Gibco (Thermo Fisher Scientific, Waltham, MA, USA). 3-BrPA, SCT and the chemotherapeutic agent 5-fluorouracil (5-FU) were purchased from Sigma-Aldrich (St. Louis, MO, USA).

Cell culture and animals. The human gastric cancer cell line SGC-7901 was purchased from the Cell Bank of the Chinese Academy of Sciences (Shanghai, China). Cells were cultured in RPMI-1640 medium supplemented with 10\% FBS, $100,000 \mathrm{U} / 1$ penicillin, and $100 \mathrm{mg} / 1$ streptomycin at $37^{\circ} \mathrm{C}$ in an incubator with $5 \% \mathrm{CO}_{2}$. The cells were harvested after trypsinization by $0.025 \%$ trypsin with $0.02 \%$ EDTA and washed twice with phosphate-buffered saline (PBS). The cells were split for further culture once they reached $\sim 80 \%$ confluency. Experiments were not conducted until the cells were in logarithmic growth phase. The 5-to-6-week old female BALB/c nude mice (weighing between 18 and $20 \mathrm{~g}$ ) were purchased from the Animal Experimental Center of Guangxi Medical University (Guangxi, China) and fed under specific pathogenfree conditions. The experimental protocol was carried out under the supervision of the Ethics Committee of Guangxi Medical University, and in accordance with internationally recognized guidelines on Animal Welfare.

Cell viability assay. Cell viability was assessed with the modified tetrazolium salt 3-(4,5-dimethylthiazol-2-yl)-2,5-diphenyltetrazolium bromide (MTT) method. Briefly, SGC-7901 cells were exposed to different concentrations of 3-BrPA or SCT (or 5-FU or the PBS control) for 24 or $48 \mathrm{~h}$ after being seeded onto 96 -well plates $(\sim 2,000$ cells/well) for $24 \mathrm{~h}$. The 3 -BrPA and SCT solutions were prepared in RPMI-1640 medium, adjusted to $\mathrm{pH} 7.4$ with $\mathrm{NaOH}$, and then sterilized using a $0.22-\mu \mathrm{m}$ filter unit (Millipore, Billerica, MA, USA). To each well, $0.01 \mathrm{ml} \mathrm{MTT}$ solution $(5 \mathrm{mg} / \mathrm{ml})$ was added and incubated at $37^{\circ} \mathrm{C}$ for $4 \mathrm{~h}$. Formazan crystals were then dissolved with DMSO, and the optical densities at $490 \mathrm{~nm}$ were measured using a microplate reader (Bio-Rad, Hercules, $\mathrm{CA}$, USA). The $\mathrm{IC}_{50}$ value (concentration of $50 \%$ inhibition) was obtained from three independent repetitive trials.

Analysis of cell morphology. SGC-7901 cells were cultured with 5 -FU $(0.5 \mathrm{mmol} / \mathrm{l})$ and different concentrations of 3 -BrPA and SCT for $24 \mathrm{~h}$. The morphological changes in cells were photographed using inverted microscopy (Nikon, Tokyo, Japan).

Analysis of cell apoptosis. The apoptosis of SGC-7901 cells was analyzed by flow cytometry (FCM) with the Annexin V:PE Apoptosis Detection kit I (BD Biosciences, USA). Briefly, the cells were collected after being treated for 24 or $48 \mathrm{~h}$ with 3-BrPA, SCT, 5-FU or the PBS control, resuspended in $100 \mu \mathrm{l}$ of binding buffer with $5 \mu \mathrm{l}$ Annexin V-PE and $5 \mu 1$ 7-aminoactinomycin D (7-AAD), washed twice with cold PBS, and then incubated in the dark at room temperature for $15 \mathrm{~min}$. The samples were then analyzed by FCM (Beckman Coulter, Miami, FL, USA).

Cell cycle assay. SGC-7901 cells were collected after being treated for $24 \mathrm{~h}$ with 3-BrPA, SCT, 5-FU or the PBS control, washed twice with PBS and fixed in $70 \%$ cold ethanol. The cells were then incubated with RNase A $(0.1 \mathrm{mg} / \mathrm{ml})$ at $37^{\circ} \mathrm{C}$ for $30 \mathrm{~min}$, and then with propidium iodine (PI, $0.2 \mathrm{mg} / \mathrm{ml})$ at $4^{\circ} \mathrm{C}$ for $60 \mathrm{~min}$ before analysis. Cell cycle progression was measured using the Cell Cycle Detection kit (Keygen, Nanjing, China) and analyzed by FCM.

Glycolytic enzyme, ATP and lactate assays. According to the manufacturer's instructions in the HK, PFK-1, PK, ATP and lactate assay kits (Jiancheng, Nanjing, China), we measured the activity or concentration of HK, PFK-1, PK, ATP and lactate spectrophotometrically (UV-2450; Shimadzu, Japan) at an absorbance of $340 \mathrm{~nm}$. Samples were obtained from SGC-7901 cells after being treated for 24 or $48 \mathrm{~h}$ with 3-BrPA, SCT, 5-FU or the PBS control. The activities of the enzymes were calibrated with cellular protein concentration.

Quantitative real-time PCR ( $R T$ - $q P C R)$ assay. Total RNA was prepared by using the total RNA Extraction kit (Axygen, Union City, CA, USA) and reverse-transcribed with the PrimeScript ${ }^{\circledR}$ RT reagent kit (Takara, Tokyo, Japan). The primers for Bax, Bcl-2, Cyt-C, survivin, and GAPDH synthesized by Sangong Biotech (Shanghai, China) are shown in Table I. The reaction was carried out using the SYBR Green PCR Master Mix (Roche, USA). Real-time PCR assays were performed using Applied Biosystems ${ }^{\circledR} 7500$ Real-Time PCR systems (Life Technologies, MA, USA) according to the manufacturer's instructions. PCR was carried out for 40 cycles of $95^{\circ} \mathrm{C}$ for 
Table I. Primers of related genes used for RT-qPCR analysis.

\begin{tabular}{lll}
\hline Genes & \multicolumn{1}{c}{ Forward primer $\left(5^{\prime}\right.$ to $\left.3^{\prime}\right)$} & Reverse primer $\left(5^{\prime}\right.$ to $\left.3^{\prime}\right)$ \\
\hline Bax & CCGATTCATCTACCCTGCTG & TGAGCAATTCCAGAGGCAGT \\
Bcl-2 & GAGGATTGTGGCCTTCTTTG & GTGCCGGTTCAGGTACTCA \\
Cyt-C & TGTCGGCATTAAGAAGAAGGA & TAAATCAGGACTGCCCAACA \\
Survivin & TCAAGGACCACCGCATCTCT & CAGTGGGGCAGTGGATGAA \\
GAPDH & GTCAGCCGCATCTTCTTT & CGCCCAATACGACCAAAT
\end{tabular}

$15 \mathrm{sec}$ and $60^{\circ} \mathrm{C}$ for $30 \mathrm{sec}$. The mRNA expression levels were calculated relative to GAPDH by using the $2^{-\Delta \Delta C T}$ method.

Western blot analysis. The total protein was extracted from the SGC-7901 cells after being treated for $24 \mathrm{~h}$ with 3-BrPA, SCT, 5-FU or the PBS control, and protein concentration was determined using the BCA method. A $15 \%$ separation gel was used to separate the proteins using SDS-PAGE at a constant voltage $(80 \mathrm{mV})$ for $90 \mathrm{~min}$. A $5 \%$ stacking gel and water bath method were used to transfer the membranes at a constant current (180 mA) for 30-75 min. The NC membrane (Millipore) was blocked with 5\% skimmed milk for $2 \mathrm{~h}$ at room temperature. The rabbit antibodies against Bcl-2, Bax, Cyt-C, survivin, GAPDH and the anti-rabbit IgG DyLight 800 conjugated antibody were purchased from Cell Signaling Technology (Boston, MA, USA). The rabbit antibody against cleaved caspase-3 (P17KD fragment) was purchased from Neomarker (USA). The primary antibodies were diluted with primary antibody dilution buffer (Beyotime, Shanghai, China) and incubated at $4{ }^{\circ} \mathrm{C}$ in the refrigerator overnight. The secondary antibodies were added to the slides, and then incubated for $90 \mathrm{~min}$ at room temperature. The gray scale values of the protein bands were determined by Odyssey software (LI-COR, Lincoln, NE, USA).

Tumor model of gastric orthotopic transplantation in nude mice. The back of the axillary region of each nude mouse was subcutaneously injected with $0.2 \mathrm{ml}\left(2 \times 10^{6} / \mathrm{ml}\right)$ of the SGC-7901 cell suspension. When the subcutaneous tumor became palpable (i.e., $\sim 5 \mathrm{~mm}$ after $\sim 2$ weeks), the tumor tissue was excised from the nude mouse, and then minced. The minced tumor tissue became the subcutaneous transplant for the next group of nude mice, and this subcutaneous transplantation was repeated for six generations. The tumor tissue from the sixth generation nude mice was minced to $1-2 \mathrm{~mm}^{3}$ in size before being orthotopic transplanted inside the seromuscular layer of greater curvature. We chose the seromuscular layer near the antrum of the greater curvature as the preferable operation site for its rich blood flow. On account of its secure wound adhesion, we used 1-2 drops of medical OB glue (32) to bind the serosal layer incision, before covering it with the greater omentum. We waited for $\sim 10 \mathrm{sec}$ for the glue to fully congeal to avoid the tumor tissue masses falling off. The tumor was allowed to grow for 2 weeks before treatment was initiated.

Mice were then randomized into 8 groups ( 8 mice/group) as follows: 3-BrPA low-dose group (3-BrPA-L, $1.85 \mathrm{mg} / \mathrm{kg} /$ day),
3-BrPA medium-dose group (3-BrPA-M, $2.23 \mathrm{mg} / \mathrm{kg} / \mathrm{day})$, 3-BrPA high-dose group (3-BrPA-H, $2.67 \mathrm{mg} / \mathrm{kg} / \mathrm{day}$ ), SCT low-dose group (SCT-L, $7.5 \mathrm{mg} / \mathrm{kg} / \mathrm{day}$ ), SCT medium-dose group (SCT-M, $15 \mathrm{mg} / \mathrm{kg} /$ day), SCT high-dose group (SCT-H, $30 \mathrm{mg} / \mathrm{kg} /$ day), 5-FU group (5-FU, $10 \mathrm{mg} / \mathrm{kg} /$ day), and control group (PBS, $10 \mathrm{ml} / \mathrm{kg} /$ day). 3-BrPA, SCT and 5-FU were dissolved in $\mathrm{PBS}$, adjusted to $\mathrm{pH} 7.4$ with $\mathrm{NaOH}$, and then sterilized with a $0.22-\mu \mathrm{m}$ filter unit. All mice were intraperitoneally injected with $0.2 \mathrm{ml}$ of the drug or PBS once per day for 4 weeks. Any changes observed in the nude mice, such as behavior, eating and excretion were monitored. The tumors were harvested after 4 weeks of treatment, and tumor volume was calculated as $0.5 \mathrm{x}$ length $\mathrm{x}$ width $\mathrm{x}$ thickness.

Terminal deoxynucleotidyl transferase-mediated dUTP nick end labeling (TUNEL) assay. Apoptosis in the tumor tissue was investigated by TUNEL staining using the In Situ Cell Death Detection kit (Roche, USA). The percentages of positive cells from total cells were considered as apoptotic indices (AI) after being counted in six different high power fields. The tumor tissue sections were observed under an IX73 inverted microscope (Olympus, Tokyo, Japan).

Transmission electron microscope (TEM) assay. The ultrastructure of the tumors was observed by TEM. The tumor tissues were fixed in $2.5 \%$ glutaraldehyde and $1 \%$ osmium tetroxide, dehydrated by graded ethanol, and embedded in Epon. Ultrathin sections were stained with $2 \%$ uranyl acetate and lead citrate, and then examined under a JEM-2000EX transmission electron microscope (Jeol, Tokyo, Japan).

Statistical analysis. All statistical analyses were performed using SPSS 17.0 software (SPSS, Chicago, IL, USA). The data are presented as the mean \pm standard deviation (SD). Differences between multiple groups were analyzed with one-way analysis of variance. Pairwise comparison was performed using the t-test, with significant differences determined as a value of $\mathrm{P}<0.05(\mathrm{P}<0.05$ vs. the control group and $\mathrm{P}<0.05$ vs. the $5-\mathrm{FU}$ group).

\section{Results}

Morphological changes induced by 3-BrPA and SCT. As shown in Fig. 1A, untreated cells attached closely to one another, and were polygonal in shape. However, the cells in the 5-FU-, 3-BrPA-, or SCT-treated groups became round or inflated, with fewer cellular contacts. 

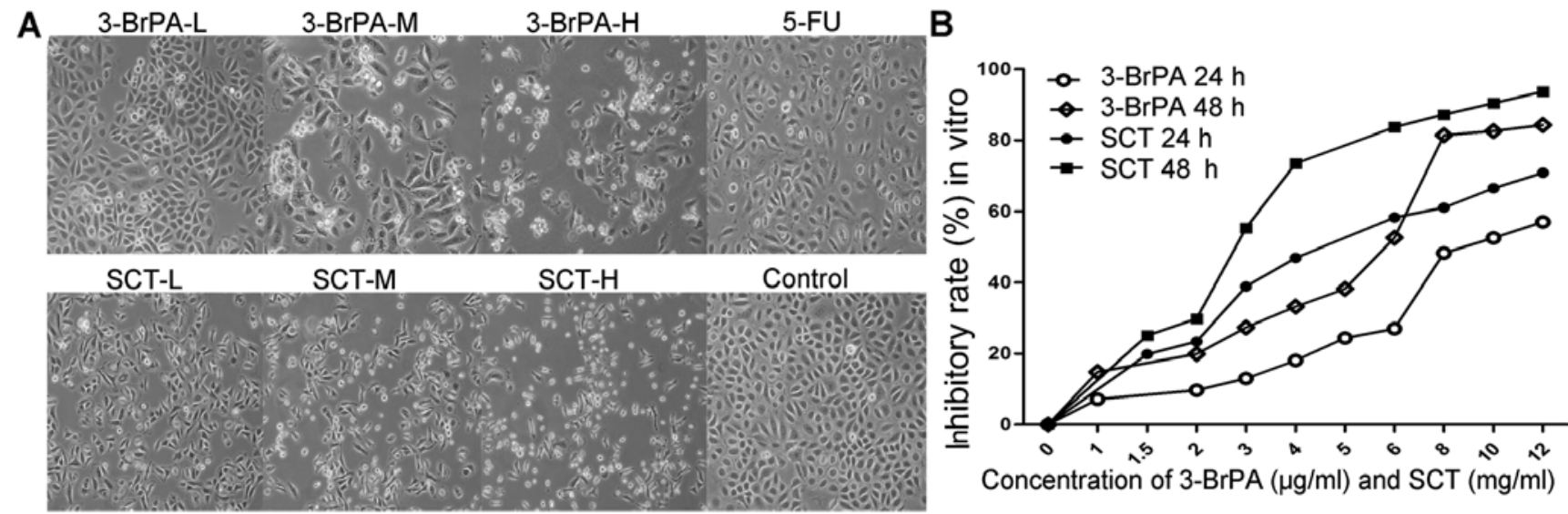
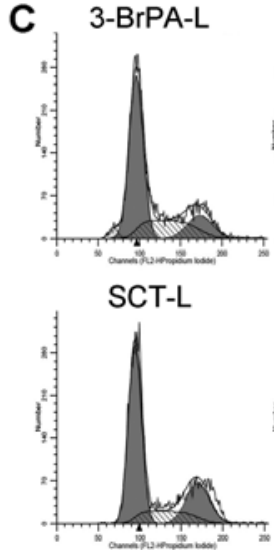

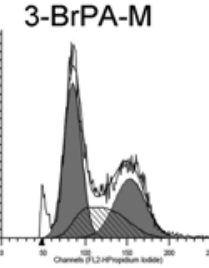

SCT-M

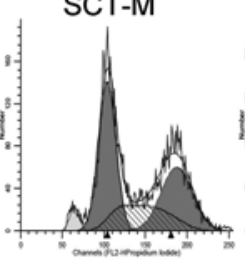

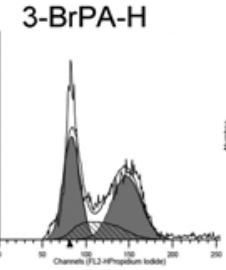

SCT-H

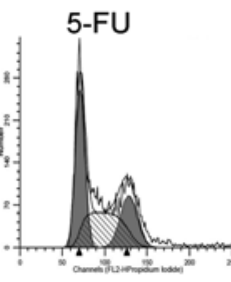

Control
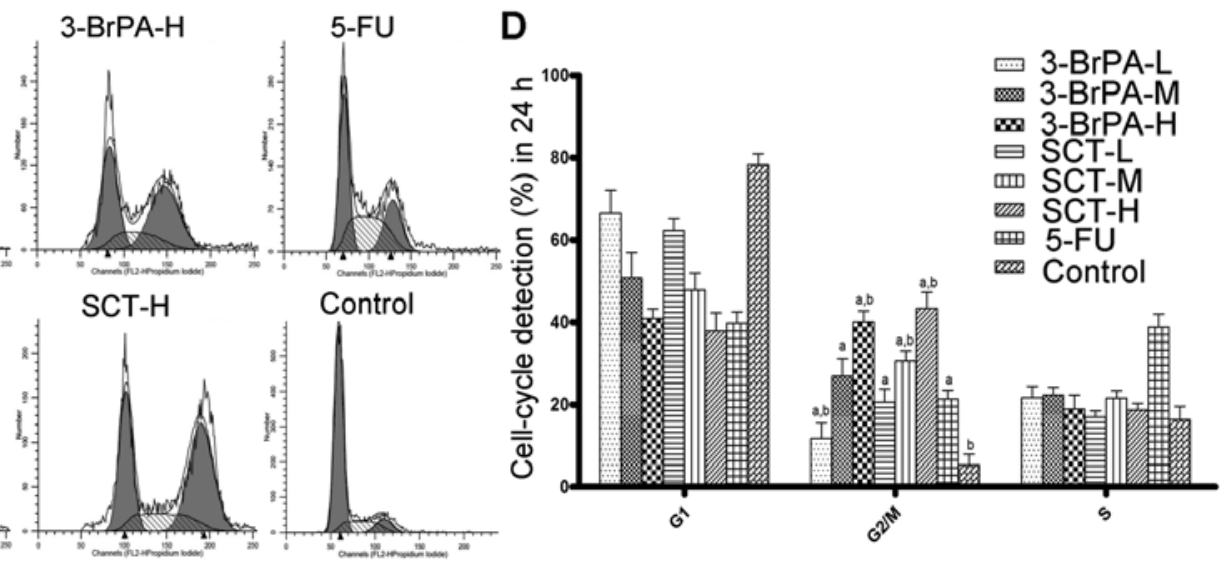

Figure 1.3-BrPA and SCT inhibit gastric cancer cell growth and induce cell cycle arrest in the G2/M phases. (A) Morphological changes were observed under an inverted microscope. Untreated cells attached closely to one another, and were polygonal in shape. However, the cells in the 5-FU, 3-BrPA or SCT groups became round or inflated with fewer cellular contacts and reduction in the amount of viable cells. (B) Cell viability was detected by MTT assay. Curves show that cell growth was inhibited by 3-BrPA and SCT in a dose- and time-dependent manner. (C and D) Cell cycle was analyzed by flow cytometry. SGC-7901 cells exposed to 3 -BrPA, SCT or 5-FU had a significantly increased cell population in the G2/M phases $\left({ }^{\mathrm{a}} \mathrm{P}<0.05\right)$.

Cell viability is reduced by $3-B r P A$ and $S C T$. We measured the proliferative effect of 3-BrPA and SCT on SGC-7901 cells using the MTT assay. As shown in Fig. 1B, we found a time- and dose-dependent decrease in cell viability after exposure to 3-BrPA or SCT. The $\mathrm{IC}_{50}$ values of 3-BrPA against SGC-7901 cells after being treated for 24 or $48 \mathrm{~h}$ were $9.88 \pm 1.07$ and $5.97 \pm 0.95 \mu \mathrm{g} / \mathrm{ml}$, respectively; however, the $\mathrm{IC}_{50}$ values of SCT were $6.47 \pm 0.87$ and $3.84 \pm 0.59 \mathrm{mg} /$ $\mathrm{ml}$. Therefore, for our in vitro study we created the following eight treatment groups: 3-BrPA low-dose group (3-BrPA-L, $4 \mu \mathrm{g} / \mathrm{ml}$ ), 3-BrPA medium-dose group (3-BrPA-M, $6 \mu \mathrm{g} / \mathrm{ml}$ ), 3-BrPA high-dose group (3-BrPA-H, $8 \mu \mathrm{g} / \mathrm{ml}$ ), SCT low-dose group (SCT-L, $1.5 \mathrm{mg} / \mathrm{ml}$ ), SCT medium-dose group (SCT-M, $3 \mathrm{mg} / \mathrm{ml}$ ), SCT high-dose group (SCT-H, $6 \mathrm{mg} / \mathrm{ml}$ ), 5-FU group (5-FU, $0.5 \mathrm{mmol} / \mathrm{l})$, and control group (untreated).

Cell cycle arrest is induced by 3-BrPA and SCT. The effect of 3-BrPA and SCT on the cell cycle was detected by FCM. As shown in Fig. 1C and D, after exposure to 3-BrPA, SCT or $5-\mathrm{FU}$ for $24 \mathrm{~h}$, the cell population in the $\mathrm{G} 2 / \mathrm{M}$ phases was significantly increased $(\mathrm{P}<0.05)$. The cell population in the $\mathrm{S}$ phase of the 5-FU group was also obviously increased $(\mathrm{P}<0.05)$. The results suggest that one of the inhibitory mechanisms of 3-BrPA or SCT is to induce cell cycle arrest to increase the rate of apoptosis.
Cell apoptosis is induced by 3-BrPA and SCT. To determine whether 3-BrPA- and SCT-induced cell death is related to apoptosis, an Annexin V:PE staining assay was conducted. As shown in Figs. 2 and 3, the percentage of apoptosis was increased from $15.60 \pm 1.83 \%$ after $24 \mathrm{~h}$ to $84.45 \pm 3.97 \%$ after $48 \mathrm{~h}$ in the 3-BrPA-treated groups, and increased from $19.31 \pm 1.89$ to $88.34 \pm 5.22 \%$ in the SCT groups. As a positive control, the percentage was increased from $61.57 \pm 4.30$ to $92.64 \pm 4.15 \%$ after treatment with $5-F U$. These increases in apoptosis percentages were significant compared with the control group $(3.24 \pm 0.72 \%$ after $24 \mathrm{~h}$ and $9.70 \pm 1.52 \%$ after $48 \mathrm{~h})(\mathrm{P}<0.05)$. The result demonstrated that dose- and time-dependent apoptosis occurs after exposure to 3-BrPA or SCT. Moreover, the reductions in cell viability using the MTT assay correlated well with the induction of apoptosis by 3-BrPA or SCT.

Regulation of glycolytic enzyme activity by 3-BrPA and SCT. We evaluated the activities of $\mathrm{HK}, \mathrm{PFK}-1$ and $\mathrm{PK}$ to investigate whether 3-BrPA and SCT kill cancer cells through regulation of glycolytic enzymes. As shown in Fig. 4A, 3-BrPA significantly reduced $\mathrm{HK}$ activity in a time- and dose-dependent manner $(\mathrm{P}<0.05)$. The HK activity observed in the 5-FU group was less than that in the 3-BrPA-L group but more than that in the 3-BrPA-M group $(\mathrm{P}<0.05)$. HK activity in the SCT groups 
A

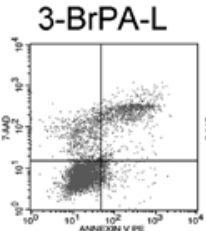

SCT-L

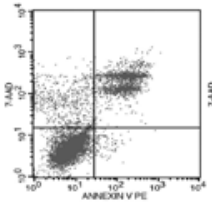

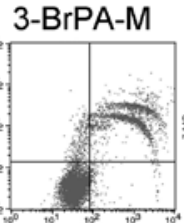

SCT-M

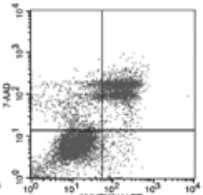

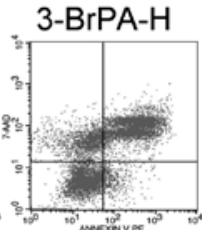

SCT-H

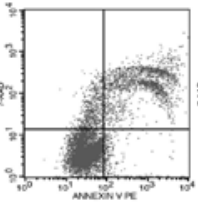

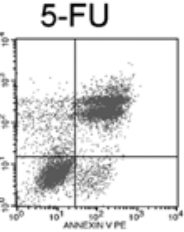

Control

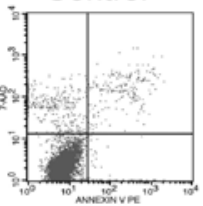

B

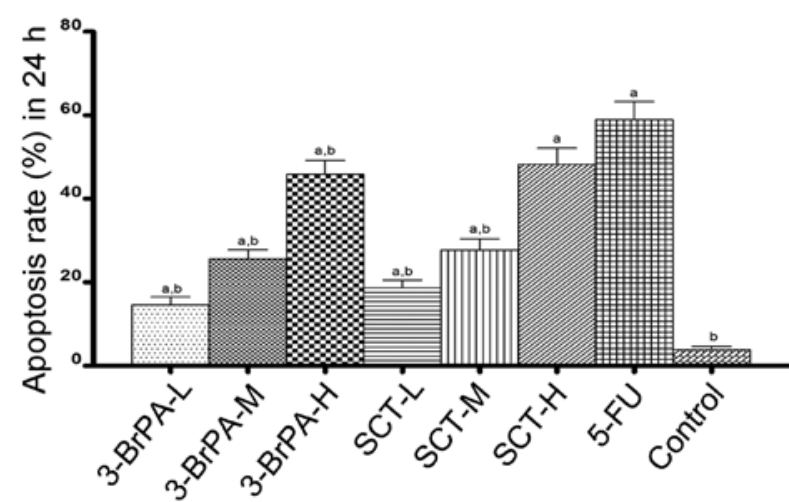

Figure 2. Cell apoptosis after $24 \mathrm{~h}$ as detected by flow cytometry. (A) The upper right quadrant shows apoptotic cells, whereas the lower left quadrant shows normal cells. (B) The percentages of apoptosis induced by 3-BrPA and SCT were markedly increased as the concentrations increased ( $\mathrm{P}<0.05$ ).

A
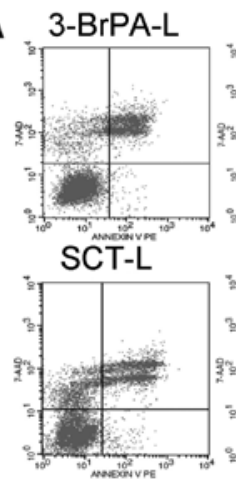

\section{3-BrPA-M}
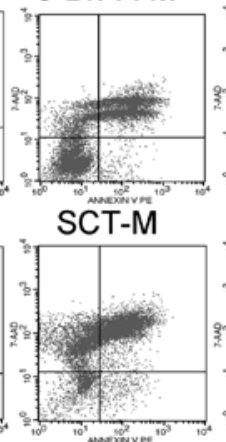
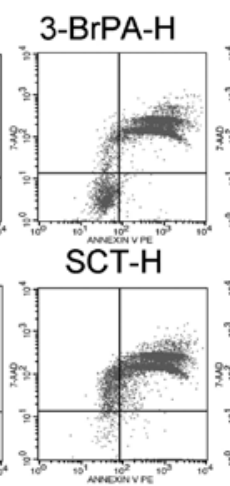

5-FU

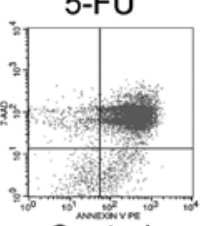

Control

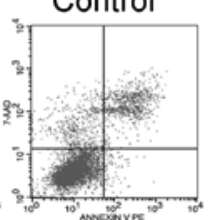

B

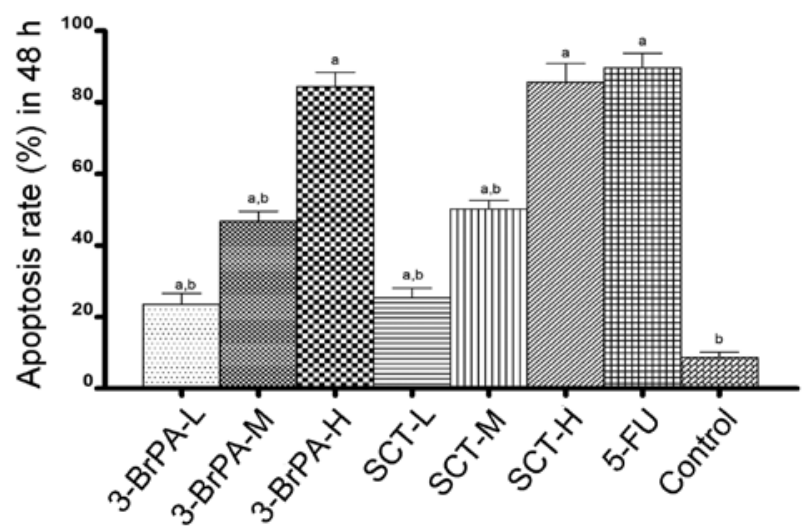

Figure 3. Cell apoptosis after $48 \mathrm{~h}$ as detected by flow cytometry. (A) The upper right quadrant shows apoptotic cells, whereas the lower left quadrant shows normal cells. (B) The percentages of apoptosis induced by 3-BrPA, SCT and 5-FU were markedly increased (aP $<0.05)$. Moreover, the results demonstrated that 3-BrPA or SCT caused apoptosis in a time- and dose-dependent manner.

had no difference when compared with the activity in the control group $(\mathrm{P}>0.05)$. As shown in Fig. 4B, PFK-1 activity decreased in a time- and dose-dependent manner in the SCT groups $(\mathrm{P}<0.05)$. However, $\mathrm{PFK}-1$ activity was not inhibited by 3-BrPA or 5-FU ( $\mathrm{P}>0.05)$. As shown in Fig. $4 \mathrm{C}$, there were no significant difference noted in PK activity in the 5-FU, 3-BrPA, and SCT groups compared with the control group $(\mathrm{P}>0.05)$.

ATP and lactate production is reduced by 3-BrPA and SCT. ATP and lactate are the main end products of glycolysis, and are regarded as efficient indicators of the glycolysis rate. As shown in Fig. 5, both the 3-BrPA and SCT groups exhibited a significant time- and dose-dependent decrease in cellular ATP levels and lactate production $(\mathrm{P}<0.05)$. Moreover, the cellular ATP and lactate levels in the 5-FU group after treatment for $8 \mathrm{~h}$ were obviously reduced compared with the control group $(\mathrm{P}<0.05)$. These results indicate that $3-\mathrm{BrPA}$ and SCT may cause apoptosis by blocking glycolysis, which is required for energy metabolism.

Expression of apoptosis-related genes is altered by 3-BrPA and SCT. To determine the effect of apoptosis-related genes induced by 3-BrPA and SCT, the mRNA levels of Bax, Bcl-2, $\mathrm{Cyt}-\mathrm{C}$ and survivin were detected by RT-qPCR analysis. As shown in Fig. 6, compared with the control group, the mRNA levels of Bax and Cyt-C were increased, while the mRNA levels of Bcl-2 and survivin were decreased after exposure to 3-BrPA, SCT, or 5-FU $(\mathrm{P}<0.05)$. Furthermore, we found that 3-BrPA or SCT produced these effects in a dose-dependent manner. The mRNA levels of Bax and Cyt-C in the SCT-H group were higher than these levels in the 3-BrPA-H group $(\mathrm{P}<0.05)$. While 5-FU also increased the mRNA expression of Bax and Cyt-C, and decreased the mRNA expression of Bcl-2, it was less effective than 3-BrPA-H and SCT-H $(\mathrm{P}<0.05)$. However, the inhibitory effect of 5-FU on survivin mRNA expression was the highest among all the groups $(\mathrm{P}<0.05)$.

Expression of apoptosis-related proteins is altered by 3-BrPA and SCT. To further confirm that 3-BrPA and SCT regulate the expression of apoptosis-related genes, we determined the protein expression of Bax, Bcl-2, Cyt-C, cleaved caspase-3, and survivin by western blot analysis. As shown in Fig. 7, 3-BrPA and SCT both upregulated Bax, Cyt-C, and cleaved caspase-3 protein expression, and downregulated Bcl-2 and survivin protein expression in a dose-dependent manner $(\mathrm{P}<0.05)$. 5-FU also increased the protein expression of Bax, Cyt-C, cleaved caspase- 3 and decreased the expression of Bcl-2 and survivin, but its ability was weaker than 3-BrPA-M and SCT-M $(\mathrm{P}<0.05)$. The increased expression of Bax, and downregulation of Bcl-2 and survivin, in the 3-BrPA-H group 
A

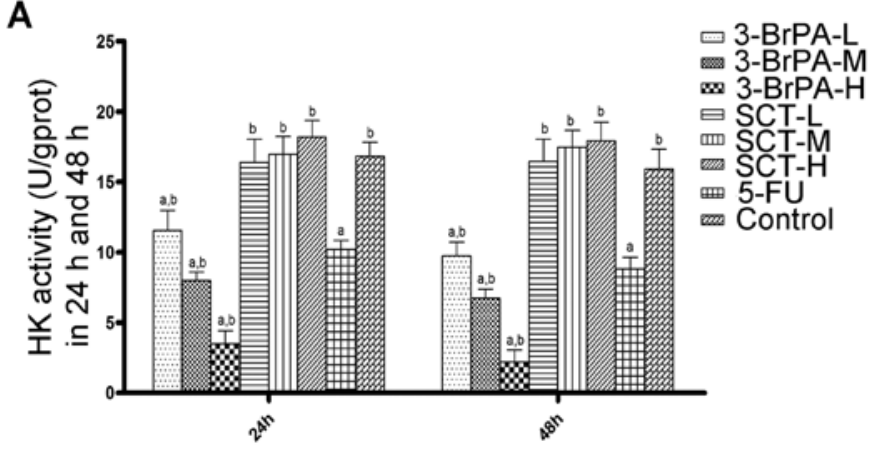

B

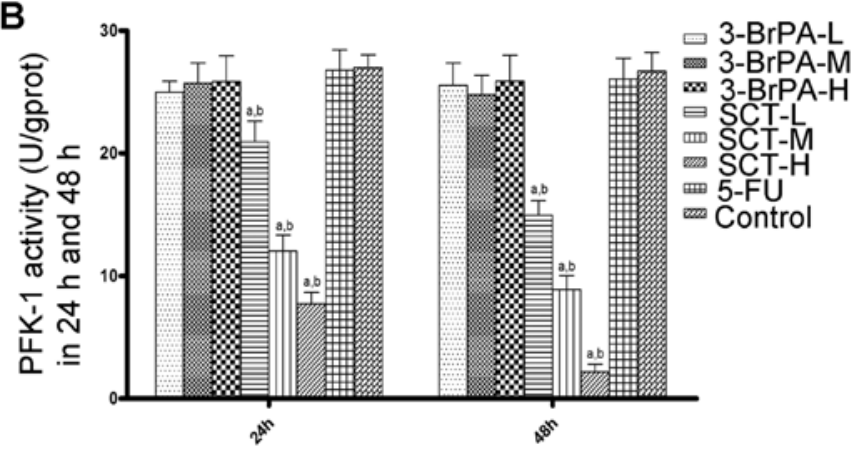

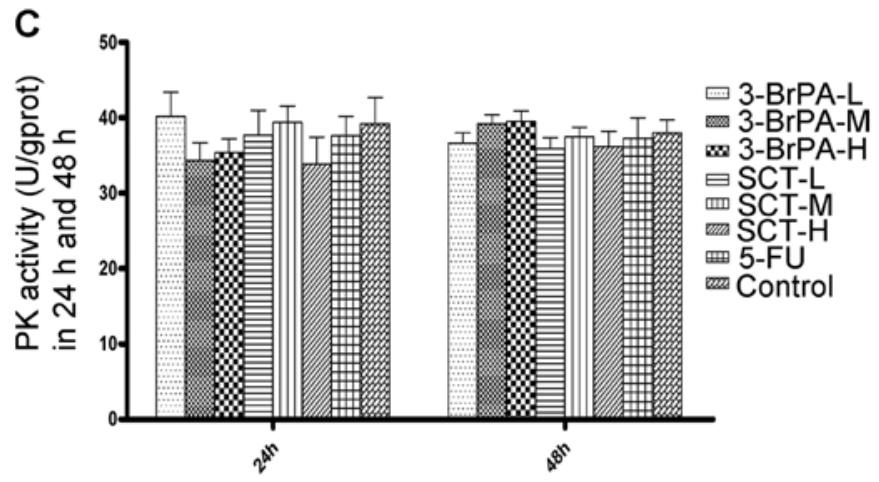

Figure 4. Activities of glycolytic enzymes. (A) HK activity was significantly reduced with 3-BrPA treatment in a time-and dose-dependent manner ( $\mathrm{P}<0.05$ ). Yet, it was not affected by SCT ( $\left.{ }^{\mathrm{P}}>0.05\right)$. (B) PFK-1 activity decreased in a time- and dose-dependent manner in the SCT groups ( $\left({ }^{\mathrm{a}, \mathrm{b}} \mathrm{P}<0.05\right)$. However, $\mathrm{PFK}-1$ activity was not suppressed by 3-BrPA and 5-FU ( $\left.{ }^{a} \mathrm{P}>0.05\right)$. (C) PK activity was not inhibited by 3-BrPA, SCT or 5-FU ( $\left.{ }^{a}>0.05\right)$.

A

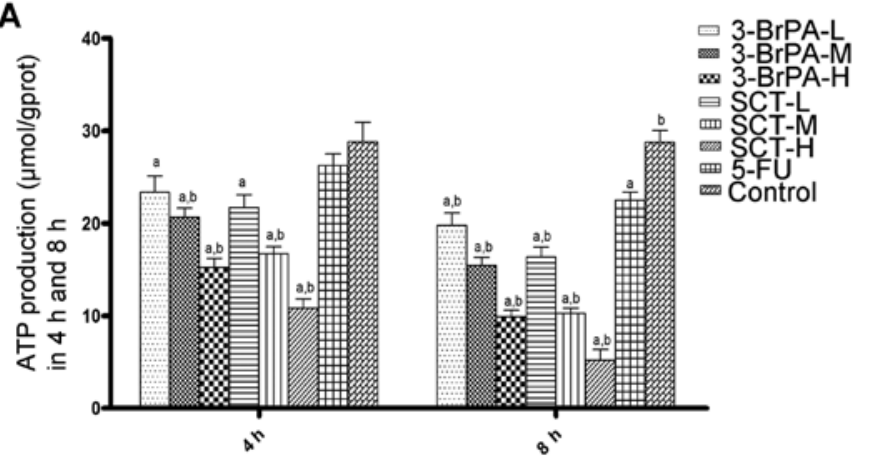

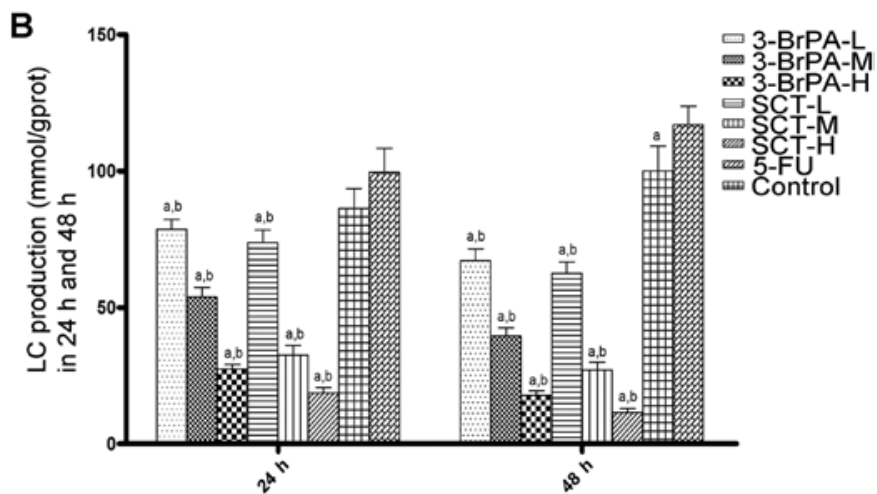

Figure 5. Production of ATP and lactate. (A) Cells treated with 3-BrPA or SCT exhibited a significant dose-and time-dependent decrease in intracellular ATP production $\left({ }^{\mathrm{a}} \mathrm{P}<0.05\right)$. (B) 3 -BrPA or $\mathrm{SCT}$ induced a decrease in intracellular lactate production in a dose- and time-dependent manner $\left({ }^{\mathrm{P}} \mathrm{P}<0.05\right)$.

was less than that in the SCT-H group $(\mathrm{P}<0.05)$. Expression levels of Cyt-C and cleaved caspase-3 in the 3-BrPA-H group were not significantly different when compared with the SCT-H group $(\mathrm{P}>0.05)$.

\section{3-BrPA and SCT inhibit gastric orthotopic transplantation} tumor growth in vivo. To determine whether 3-BrPA and SCT are effective against gastric tumors in vivo, we produced a gastric orthotopic transplantation tumor model and administered an intraperitoneal injection of 3-BrPA, SCT, 5-FU or PBS for 4 weeks. As shown in Fig. 8A, models of human gastric orthotopic transplantation tumors in nude mice were successfully made, and the tumor formation rate was $85 \%$. As shown in Fig. 8A and B, tumor volumes in the 3-BrPA-, SCT-, or 5-FU-treated mice were significantly reduced compared with the PBS-treated mice $(\mathrm{P}<0.05)$. Furthermore, 3-BrPA and SCT inhibited the orthotopic transplantation tumor growth in a dose-dependent manner $(\mathrm{P}<0.05)$.

3-BrPA and SCT induce apoptosis in the gastric orthotopic transplantation tumors. We next evaluated whether 3-BrPA and SCT induce apoptosis in orthotopic transplantation tumors by TUNEL staining. As shown in Fig. 8C, a greater proportion of apoptotic cells, appearing with brownish granules in the cytoplasm and nucleus, were observed in the 3-BrPA, $\mathrm{SCT}$, and 5-FU groups. As shown in Fig. 8D, the apoptosis 


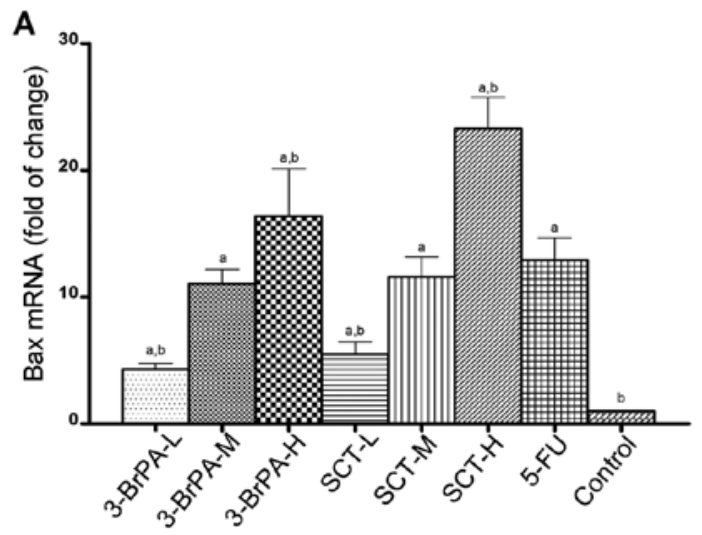

B
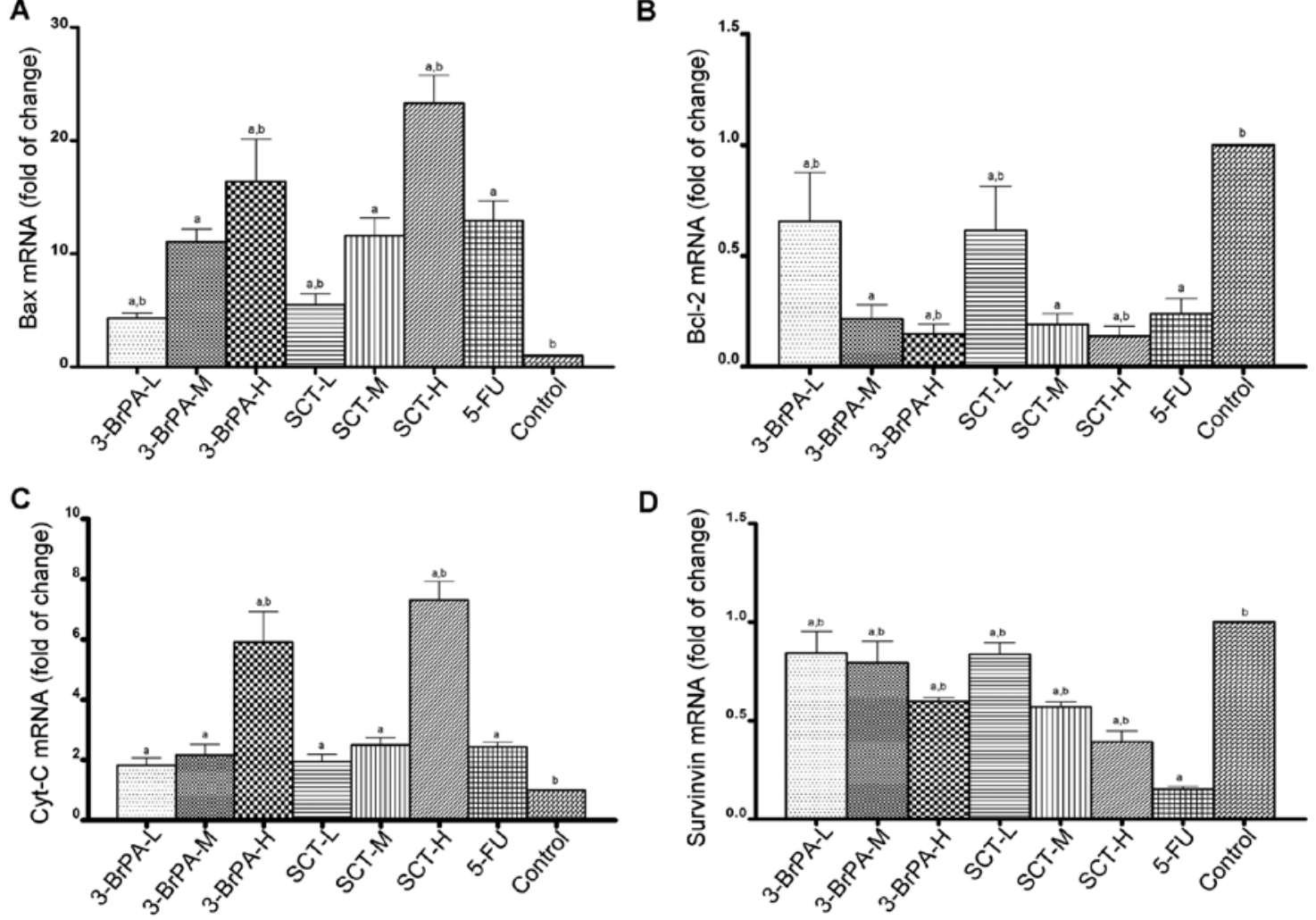

D

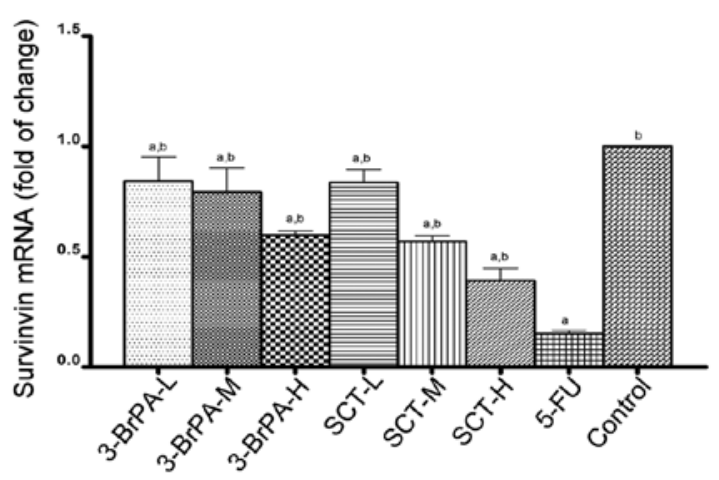

Figure 6. Expression of apoptosis-related genes as detected by RT-qPCR. (A-D) The mRNA levels of Bax, Bcl-2, Cyt-C and survivin were detected by RT-qPCR. The mRNA expression of Bax and Cyt-C were increased and Bcl-2 and survivin were decreased after exposured to 3-BrPA, SCT or 5-FU ( ${ }^{\mathrm{P}}<0.05$ ). Furthermore, we found that 3-BrPA and SCT caused these changes in a dose-dependent manner.

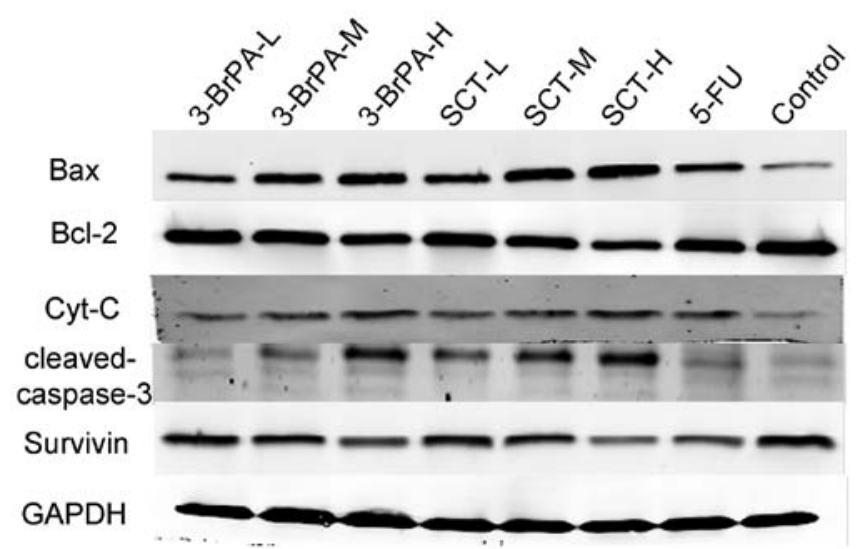

Figure 7. Expression of apoptosis-related proteins as assessed by western blot analysis. The protein expression levels of Bcl-2, Bax, survivin, Cyt-C and cleaved caspase-3 were detected by western blot analysis. 3-BrPA and $\mathrm{SCT}$ upregulated Bax, Cyt-C and cleaved caspase-3 protein expression and downregulated Bcl-2 and survivin protein expression in dose-dependent manner $\left({ }^{\mathrm{a}} \mathrm{P}<0.05\right)$.

index (AI) in each group was as follows: 3-BrPA-L group $12.50 \pm 2.43 \%$; 3 -BrPA-M group $26.29 \pm 5.76 \%$; 3-BrPA-H group $52.21 \pm 6.92 \%$; SCT-L group $13.98 \pm 2.28 \%$; SCT-M group $30.93 \pm 3.79 \%$; SCT-H group 55.07 $\pm 7.38 \%$; 5-FU group $52.71 \pm 6.73 \%$; and control group $3.29 \pm 0.76 \%$. A significant difference in AI values was identified between the 3-BrPA-, SCT-, or 5-FU-treated group and the control group $(\mathrm{P}<0.05)$. The AI of the 5-FU group was similar to the 3-BrPA-H and
SCT-H groups (P>0.05). Furthermore, 3-BrPA-H and SCT-H both induced apoptosis of the orthotopic transplantation tumors in a dose-dependent manner.

3-BrPA and SCT induce ultrastructure changes in the tumors typical of apoptosis. To further confirm the apoptotic effects, ultrastructural changes in the tumors treated by $3-\mathrm{BrPA}, \mathrm{SCT}$, or 5-FU were observed by TEM. As shown in Fig. 9, typical features of apoptosis, such as the formation of apoptotic bodies, mitochondrial crest fracture, or disappearance and chromatin concentration or fragmentation, were observed in the 3-BrPA, SCT and 5-FU groups. However, the control group had large nuclei, with prominent nucleoli, and no apoptotic bodies were observed.

\section{Discussion}

In the present study, we explored the mechanisms of 3-BrPAand SCT-mediated inhibition of in vitro human gastric cancer cell growth, and in vivo gastric orthotopic transplantation tumor growth in nude mice. We found that 3-BrPA and SCT effectively suppressed cancer cell proliferation, arrested the cell cycle in the G2/M phases, induced apoptosis, and decreased the production of lactate and ATP, which indicates inhibition of glycolysis. Moreover, 3-BrPA significantly reduced the activity of the glycolytic enzyme HK, while SCT selectively inhibited the activity of the glycolytic enzyme PFK-1 in a time- and dose-dependent manner. Furthermore, 3-BrPA and SCT upregulated Bax, Cyt-C, and cleaved 

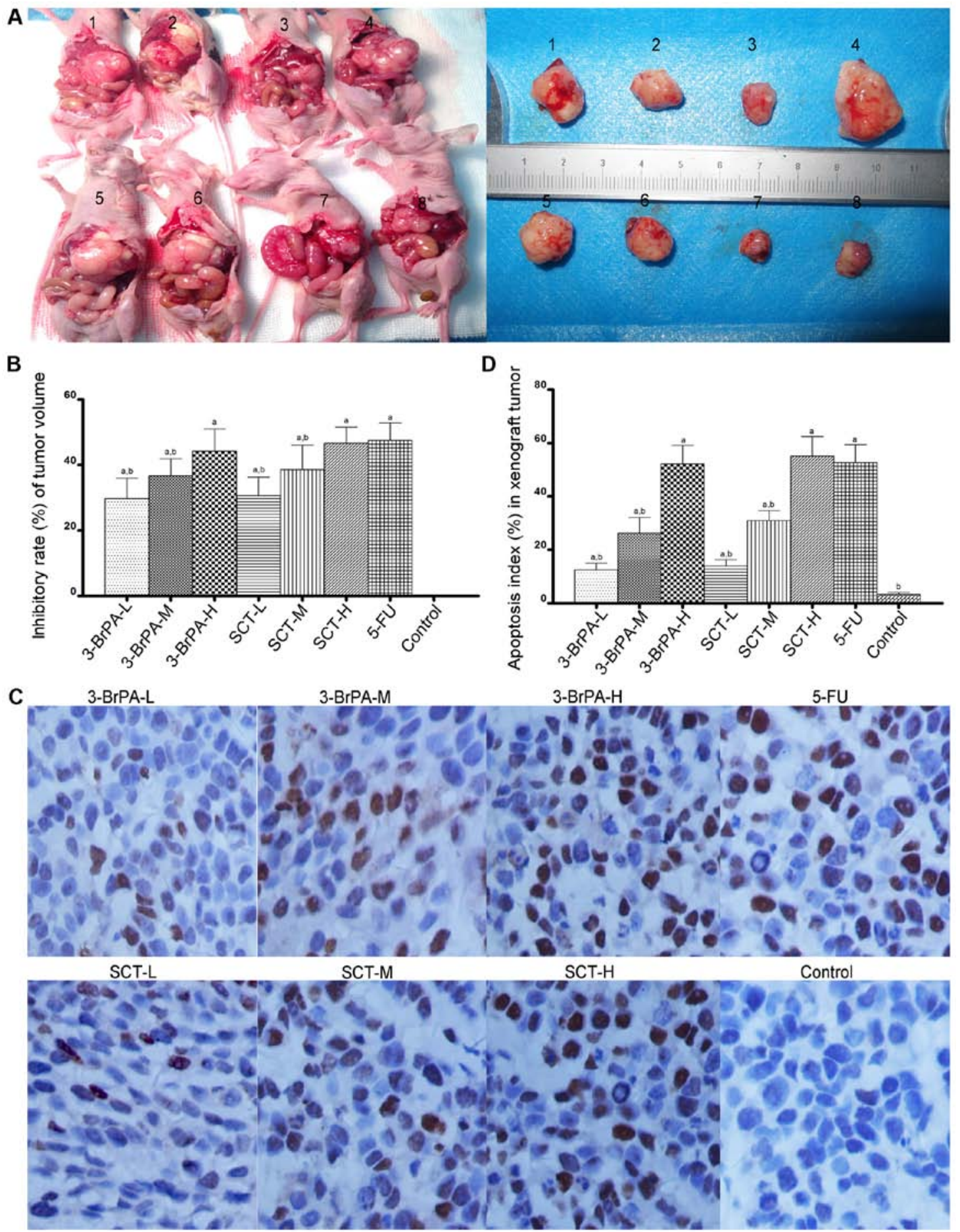

Figure 8. 3-BrPA and SCT mediate the inhibition of gastric orthotopic transplantation tumor growth and induction of apoptosis. (A) The tumor model of gastric orthotopic transplantation in nude mice was successfully constructed. Image 1, 3-BrPA-L group; 2, 3-BrPA-M group; 3, 3-BrPA-H group; 4, control (PBS) group; 5, SCT-L group; 6, SCT-M group; 7, SCT-H group; 8, 5-FU group. (A and B) After an intraperitoneal injection of 3-BrPA, SCT or 5-FU for 4 weeks, tumor volumes in the 3-BrPA-, SCT- or 5-FU-treated mice were significantly reduced ( $\left.{ }^{\mathrm{a}} \mathrm{P}<0.05\right)$. (C) Images of TUNEL revealing apoptotic cells which appeared as brownish granules in the cytoplasm and nucleus. A greater proportion of apoptotic cells was observed in the 3-BrPA, SCT and 5-FU groups (magnification, $\mathrm{x} 400$ ). (D) A significant difference in AI values was noted between the 3-BrPA-, SCT- or 5-FU-treated group and the PBS-treated group $\left({ }^{2} \mathrm{P}<0.05\right)$.

caspase-3, but downregulated Bcl-2 and survivin mRNA and protein expression. Finally, our in vivo animal study indicated that intraperitoneal injections of 3-BrPA and SCT suppressed orthotopic transplantation tumor growth and induced tumor apoptosis.

While glycolysis and apoptosis have previously been regarded as independent pathways $(30,31)$, our results indi- cated that cell apoptosis and cell cycle arrest were closely associated with glycolytic enzyme inhibition. 3-BrPA significantly reduced the activity of the glycolytic enzyme HK. By interacting with the outer membrane protein voltage dependent anion channel (VDAC), HK can stop the release of proteins from the mitochondrial intermembrane space, including Bax and Cyt-C. Thus, HK can enhance cell proliferation and 

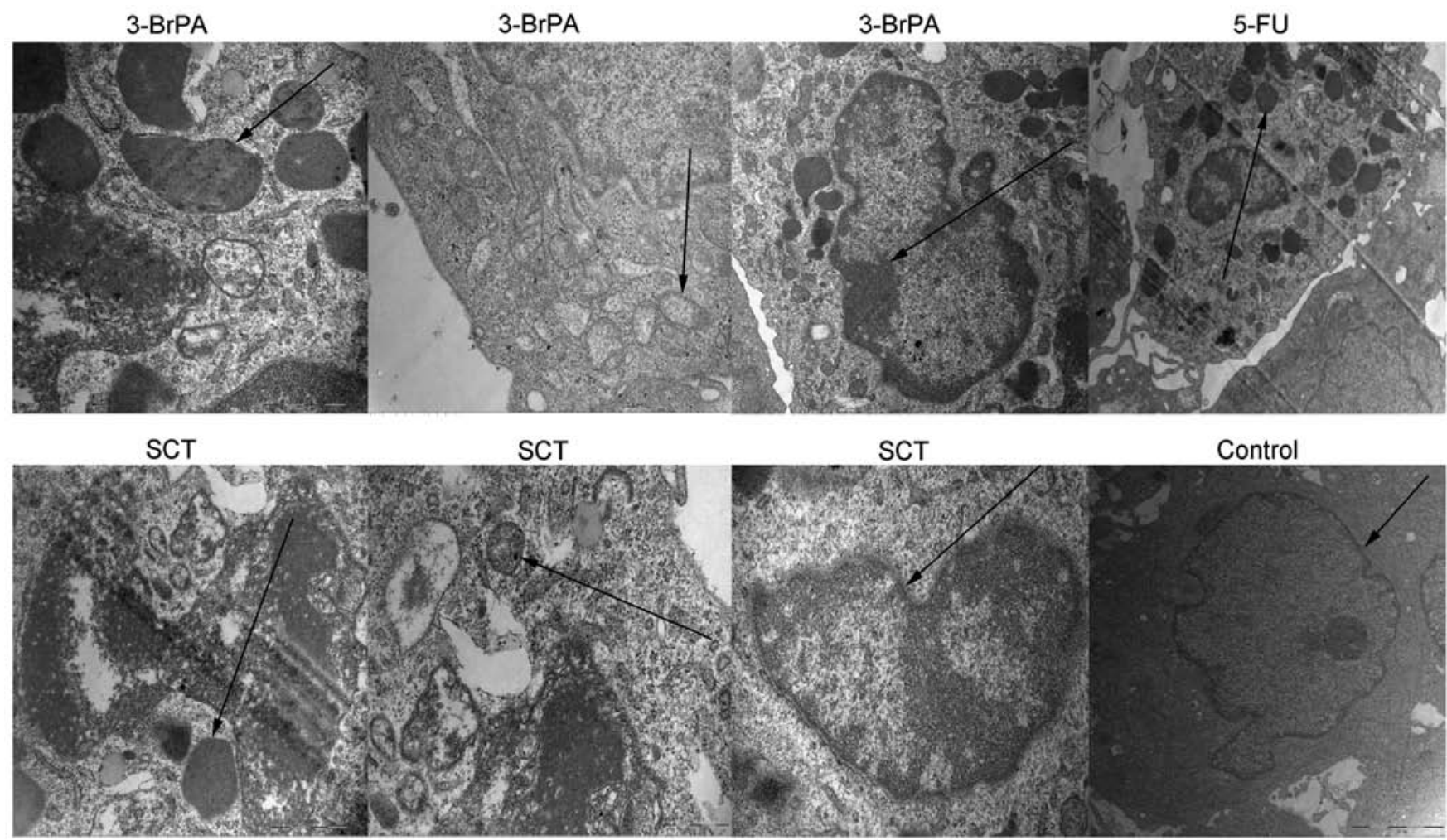

Figure 9. 3-BrPA and SCT mediate changes in tumor ultrastructure as observed by TEM. Typical features of apoptosis such as formation of apoptotic bodies, mitochondrial crest fracture or disappearance and chromatin concentration or fragmentation were observed in the 3-BrPA, SCT or 5-FU groups. However, the PBS-treated group had large nuclei with prominent nucleoli and no apoptotic bodies were observed.

suppress apoptosis by binding to mitochondria (14). We speculate that 3-BrPA inhibits $\mathrm{HK}$ activity and isolates it from the mitochondria, allowing the VDAC to open, and release $\mathrm{Cyt}-\mathrm{C}$, thereby inducing caspase-mediated apoptosis. Therefore, there is a link between glycolysis and the mitochondrial apoptotic pathway, which can both be targeted by 3-BrPA and/or SCT.

We also found that 3-BrPA and SCT downregulated survivin expression. Survivin specifically binds caspase-3, caspase-7, and caspase-9, and inhibits their activity to suppress apoptosis (33). Therefore, by downregulating survivin expression, apoptosis was no longer suppressed in the gastric cancer cells. This result suggests that 3-BrPA and SCT have multiple mechanisms by which they promote apoptosis and prevent cell proliferation in gastric tumors.

Next, we determined whether 3-BrPA and SCT were effective against gastric tumors in vivo, by using a gastric orthotopic transplantation tumor model in nude mice and monitoring the tumor volume inhibition rate and tumor apoptosis. Our data showed that 3-BrPA and SCT suppressed the growth and induced the apoptosis of human gastric cancer tumors in vivo. Moreover, we determined that intraperitoneal injection is an effective form of administration of 3-BrPA and SCT.

In conclusion, the present study identified that cell cycle arrest, glycolytic enzyme inhibition, decreased ATP production, mitochondrial apoptotic pathway activation, and survivin suppression may be the mechanisms by which 3-BrPA and SCT inhibit proliferation and induce apoptosis in gastric cancer cells. Furthermore, intraperitoneal injections of 3-BrPA and SCT into our mouse model of gastric cancer suppressed tumor growth and induced apoptosis in vivo. However, whether selectively targeting glycolysis to exhaust ATP availability and induce apoptosis also increases cancer cell sensitivity to radiation and chemotherapy requires further investigation.

\section{Acknowledgements}

This research was supported by the National Natural Science Foundation of China (grant no. 81260366) and Guangxi Scientific Research and Technology Development Project.

\section{References}

1. Yamamoto M, Sakaguchi Y, Matsuyama A, Yoshinaga K, Tsutsui $\mathrm{S}$ and Ishida T: Surgery after preoperative chemotherapy for patients with unresectable advanced gastric cancer. Oncology 85: 241-247, 2013.

2. Corso S, Ghiso E, Cepero V, Sierra JR, Migliore C, Bertotti A, Trusolino L, Comoglio PM and Giordano S: Activation of HER family members in gastric carcinoma cells mediates resistance to MET inhibition. Mol Cancer 9: 121, 2010.

3. Vander Heiden MG, Cantley LC and Thompson CB: Understanding the Warburg effect: The metabolic requirements of cell proliferation. Science 324: 1029-1033, 2009.

4. Warburg O: The Metabolism of Tumors. Constable Press, London, 1930.

5. Liu X, Wang X, Zhang J, Lam EK, Shin VY, Cheng AS, Yu J, Chan FK, Sung JJ and Jin HC: Warburg effect revisited: An epigenetic link between glycolysis and gastric carcinogenesis. Oncogene 29: 442-450, 2010.

6. Lu Y, Zhang X, Zhang H, Lan J, Huang G, Varin E, Lincet H, Poulain L and Icard P: Citrate induces apoptotic cell death: A promising way to treat gastric carcinoma? Anticancer Res 31: 797-805, 2011. 
7. Xian SL, Wei C and Lu YF: 3 BrPA inhibits proliferation of human gastric cancer cell. Chin Pract Med J 12: 78-82, 2013. (In Chinese).

8. Liu L, Gong L, Zhang Y and Li N: Glycolysis in Panc-1 human pancreatic cancer cells is inhibited by everolimus. Exp Ther Med 5: 338-342, 2013.

9. Khatri S, Yepiskoposyan H, Gallo CA, Tandon P and Plas DR: FOXO3a regulates glycolysis via transcriptional control of tumor suppressor TSC1. J Biol Chem 285: 15960-15965, 2010.

10. Floridi A, Paggi MG and Fanciulli M: Modulation of glycolysis in neuroepithelial tumors. J Neurosurg Sci 33: 55-64, 1989.

11. Nwagwu M and Opperdoes FR: Regulation of glycolysis in Trypanosoma brucei: Hexokinase and phosphofructokinase activity. Acta Trop 39: 61-72, 1982.

12. Okar DA and Lange AJ: Fructose-2,6-bisphosphate and control of carbohydrate metabolism in eukaryotes. Biofactors 10: 1-14, 1999.

13. Rider MH, Bertrand L, Vertommen D, Michels PA, Rousseau GG and Hue L: 6-phosphofructo-2-kinase/fructose-2,6-bisphosphatase: Head-to-head with a bifunctional enzyme that controls glycolysis. Biochem J 381: 561-579, 2004.

14. Gottlob K, Majewski N, Kennedy S, Kandel E, Robey RB and Hay N: Inhibition of early apoptotic events by Akt/PKB is dependent on the first committed step of glycolysis and mitochondrial hexokinase. Genes Dev 15: 1406-1418, 2001.

15. Pelicano H, Martin DS, Xu RH and Huang P: Glycolysis inhibition for anticancer treatment. Oncogene 25: 4633-4646, 2006.

16. Vander Heiden MG, Plas DR, Rathmell JC, Fox CJ, Harris MH and Thompson CB: Growth factors can influence cell growth and survival through effects on glucose metabolism. Mol Cell Biol 21: 5899-5912, 2001

17. Wang X: The expanding role of mitochondria in apoptosis. Genes Dev 15: 2922-2933, 2001.

18. Fiandalo MV and Kyprianou N: Caspase control: Protagonists of cancer cell apoptosis. Exp Oncol 34: 165-175, 2012.

19. Cohen GM: Caspases: The executioners of apoptosis. Biochem J 326: 1-16, 1997.

20. Grosse J, Warnke E, Wehland M, Pietsch J, Pohl F, Wise P, Magnusson NE, Eilles C and Grimm D: Mechanisms of apoptosis in irradiated and sunitinib-treated follicular thyroid cancer cells. Apoptosis 19: 480-490, 2014.

21. Brunelle JK and Letai A: Control of mitochondrial apoptosis by the Bcl-2 family. J Cell Sci 122: 437-441, 2009.
22. Kim H, Rafiuddin-Shah M, Tu HC, Jeffers JR, Zambetti GP, Hsieh JJ and Cheng EH: Hierarchical regulation of mitochondrion-dependent apoptosis by BCL-2 subfamilies. Nat Cell Biol 8: 1348-1358, 2006

23. Yang J, Liu X, Bhalla K, Kim CN, Ibrado AM, Cai J, Peng TI, Jones DP and Wang X: Prevention of apoptosis by Bcl-2: Release of cytochrome c from mitochondria blocked. Science 275: 1129-1132, 1997.

24. Swanton E, Savory P, Cosulich S, Clarke P and Woodman P: Bcl-2 regulates a caspase-3/caspase-2 apoptotic cascade in cytosolic extracts. Oncogene 18: 1781-1787, 1999.

25. Altieri DC: Survivin and IAP proteins in cell-death mechanisms. Biochem J 430: 199-205, 2010

26. Ambrosini G, Adida C and Altieri DC: A novel anti-apoptosis gene, survivin, expressed in cancer and lymphoma. Nat Med 3: 917-921, 1997.

27. Ko YH, Pedersen PL and Geschwind JF: Glucose catabolism in the rabbit VX2 tumor model for liver cancer: Characterization and targeting hexokinase. Cancer Lett 173: 83-91, 2001.

28. Pedersen PL: 3-Bromopyruvate (3BP) a fast acting, promising, powerful, specific, and effective 'small molecule' anti-cancer agent taken from labside to bedside: Introduction to a special issue. J Bioenerg Biomembr 44: 1-6, 2012.

29. Shoshan MC: 3-Bromopyruvate: Targets and outcomes. J Bioenerg Biomembr 44: 7-15, 2012.

30. Killion JJ, Radinsky R and Fidler IJ: Orthotopic models are necessary to predict therapy of transplantable tumors in mice. Cancer Metastasis Rev 17: 279-284, 1998-1999.

31. Bibby MC: Orthotopic models of cancer for preclinical drug evaluation: Advantages and disadvantages. Eur J Cancer 40: $852-857,2004$

32. Shi J, Wei PK, Zhang S, Qin ZF, Li J, Sun DZ, Xiao Y, Yu ZH, Lin HM,Zheng GJ, et al: OB glue paste technique for establishing nude mouse human gastric cancer orthotopic transplantation models. World J Gastroenterol 14: 4800-4804, 2008.

33. Li F, Ambrosini G, Chu EY, Plescia J, Tognin S, Marchisio PC and Altieri DC: Control of apoptosis and mitotic spindle checkpoint by survivin. Nature 396: 580-584, 1998. 